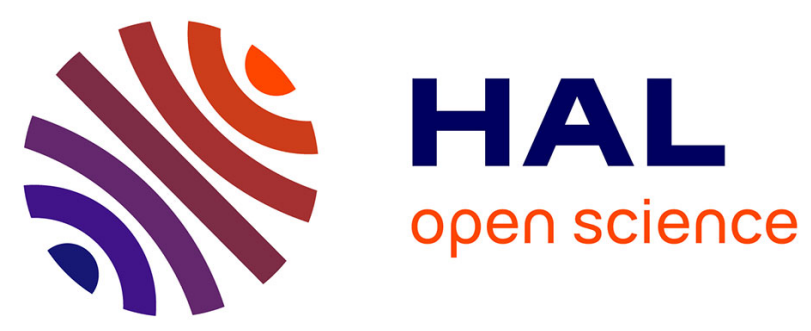

\title{
Simulating Supercapacitors: Can We Model Electrodes As Constant Charge Surfaces?
}

Céline Merlet, Clarisse Péan, Benjamin Rotenberg, Paul A Madden, Patrice Simon, Mathieu Salanne

\section{- To cite this version:}

Céline Merlet, Clarisse Péan, Benjamin Rotenberg, Paul A Madden, Patrice Simon, et al.. Simulating Supercapacitors: Can We Model Electrodes As Constant Charge Surfaces?. Journal of Physical Chemistry Letters, 2013, 4 (2), pp.264-268. 10.1021/jz3019226 . hal-00854038

\section{HAL Id: hal-00854038 https://hal.sorbonne-universite.fr/hal-00854038}

Submitted on 16 Nov 2018

HAL is a multi-disciplinary open access archive for the deposit and dissemination of scientific research documents, whether they are published or not. The documents may come from teaching and research institutions in France or abroad, or from public or private research centers.
L'archive ouverte pluridisciplinaire HAL, est destinée au dépôt et à la diffusion de documents scientifiques de niveau recherche, publiés ou non, émanant des établissements d'enseignement et de recherche français ou étrangers, des laboratoires publics ou privés. 


\section{Simulating Supercapacitors: Can we Model}

\section{Electrodes as Constant Charge Surfaces?}

Céline Merlet, ${ }^{\dagger, \ddagger}$ Clarisse Péan, $,{ }^{\dagger}, \ddagger$ Benjamin Rotenberg, ${ }^{\dagger,}$ Paul A. Madden,,$\S$ Patrice Simon, ${ }^{\mathscr{T}, \ddagger}$ and Mathieu Salanne ${ }^{*, \dagger, \ddagger}$

UPMC Université Paris 06, CNRS, ESPCI, UMR 7195, PECSA, F-75005, Paris, France, Réseau sur le Stockage Electrochimique de l'Energie (RS2E), FR CNRS 3459, France, CIRIMAT, UMR CNRS 5085, Université Paul Sabatier, Bat. 2R1, 118 route de Narbonne, 31062 Toulouse Cedex

9, France, and Department of Materials, University of Oxford, Parks Road, Oxford OXI 3PH, UK

E-mail: mathieu.salanne@upmc.fr

${ }^{*}$ To whom correspondence should be addressed

${ }^{\dagger} \mathrm{UPMC}$

RS2E

IUniversité Paul Sabatier

${ }^{\S}$ University of Oxford 


\begin{abstract}
Supercapacitors based on an ionic liquid electrolyte and graphite or nanoporous carbon electrodes are simulated using molecular dynamics. We compare a simplified electrode model in which a constant, uniform charge is assigned to each carbon atom, with a realistic model in which a constant potential is applied between the electrodes (the carbon charges are allowed to fluctuate). We show that the simulations performed with the simplified model do not provide a correct description of the properties of the system. Firstly, the structure of the adsorbed electrolyte is partly modified. Secondly, dramatic differences are observed for the dynamics of the system during transient regimes. In particular, upon application of a constant applied potential difference, the increase in the temperature, due to Joule effect, associated with the creation of an electric current across the cell follows Ohm's law, while unphysically high temperatures are rapidly observed when constant charges are assigned to each carbon atom.
\end{abstract}

Supercapacitors are used for the electrochemical storage of energy. Unlike Li-ion batteries, there is no redox process involved in the bulk of an active material, and the charge is accumulated through the electrosorption of ions from an electrolyte on the electrode surface, which confers on supercapacitors very specific properties. ${ }^{1}$ First, they show very high power densities and are therefore used in applications where there is a need for a large charge uptake or delivery in a limited amount of time (typically a few seconds). Second, only a slight volume change $(<3 \%)$ occurs in the material during operation and the electrolyte is not subject to electrochemical degradation, and thus most devices show an outstanding cycle life ( $>1,000,000$ cycles). They have nevertheless an inherently moderate energy density, and there is an increased need for higher-energy devices. To this end a better understanding of the electrolyte/electrode interface is required, for which molecular simulations appear as a promising tool, ${ }^{2-6}$ complementary to electrochemical ${ }^{7,8}$ or spectroscopic ${ }^{9,10}$ techniques.

At such an electrified interface, electrostatic interactions play a predominant role. The objective of this work is to study the importance of accounting realistically for electrode polarisation effects in the presence of an electrolyte. To this end, we perform a first series of simulations in which we 
impose that the potential on each electrode atom at each molecular dynamics step is constant and equal to a specified value ${ }^{11} V_{0}^{i}$, which is the electrode potential in a conventional electrochemistry experiment $^{12}\left(V_{0}^{i}=V^{+}\right.$or $V^{-}$for atoms in the positive and negative electrodes, respectively, with the potential difference equal to $V^{+}-V^{-}$). In practice, this is done by treating the partial charges carried by the electrode carbon atoms as additional degrees of freedom, which fluctuate during the simulation. Their values are determined from a self-consistent calculation; this approach has now been used in several simulation studies $3,5,6,12-16$. Were this electrostatic response found negligible, a significant gain of simulation time would be obtained. In order to assess the importance of these effects, simulations performed at constant applied potential are compared here with a second series of simpler, computationally less expensive simulations where fixed partial charges are assigned on each atom, as reported in a majority of simulation studies. ${ }^{17-26}$ In both cases, the same coarsegrained model is used to describe the electrolyte. ${ }^{15,27}$ We show that the simplified simulation scheme does not reproduce well a realistic electrode, both from the structural and the dynamic point of view.

In a first step we have focused on a supercapacitor simulation cell based on a 1-butyl-3methylimidazolium hexafluorophosphate $\left(\mathrm{BMIM}_{\mathrm{P}} \mathrm{PF}_{6}\right)$ ionic liquid electrolyte and graphite electrodes. This system was chosen because it is well known ${ }^{15,27}$ and because the planar surface of the electrodes facilitates the analysis of the adsorbed electrolyte structure. In order to perform a fair comparison, the constant charges were assigned based on the average total charge measured during constant potential simulations for various applied potential differences (see the Supplementary Information). This total charge was equally distributed among the carbon atoms, and the selfconsistency of the procedure was checked by calculating the potential difference arising from the so-built constant charge simulations: The initial values were well reproduced.

The ionic density profiles along the normal to the graphite surfaces are shown on Figure 1. On each electrode, only the profiles corresponding to the oppositely charged ions are shown for clarity. The region corresponding to $z$ values between 3.0 and $9.3 \mathrm{~nm}$ is not shown because it corresponds to the bulk for which ionic densities remain flat under all conditions. Although the constant poten- 

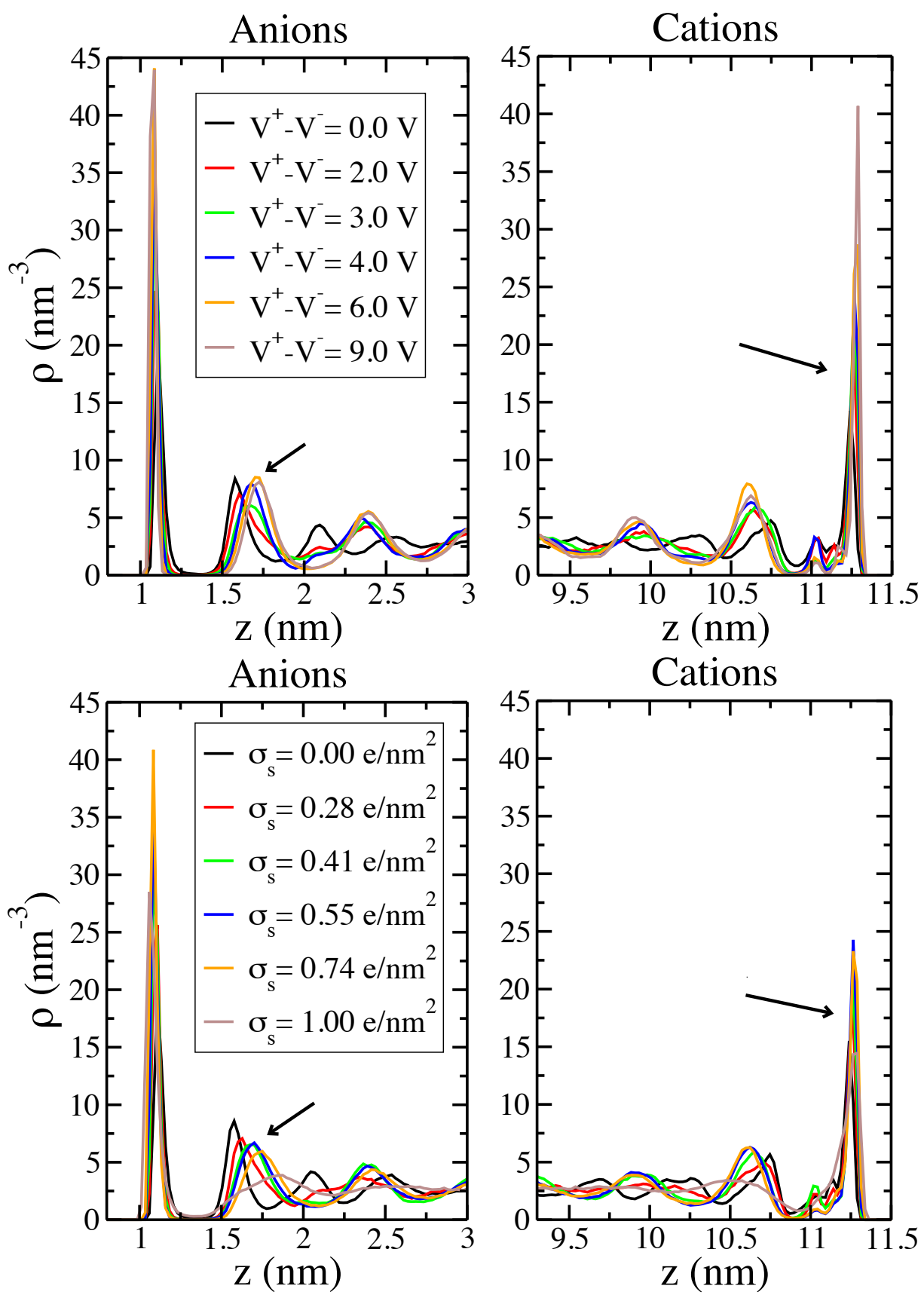

Figure 1: Ionic density profiles for the constant applied potential (top) and constant charges (bottom) simulations. For each electrode, only the oppositely charged ions profiles are shown for clarity. The arrows highlight the main differences between the two sets of data, which are discussed in the text.

tial simulations (top panel) profiles look very similar to the constant charge ones (bottom panel) at first glance, some differences are observed for both electrodes. Indeed, for the positive electrode 
(left panels), the second peak of the $\mathrm{PF}_{6}^{-}$ions density profiles, located at distances between 1.5 and $2.0 \mathrm{~nm}$, shows a different variation depending on the simulation scheme. In the first case, the intensity remains constant whatever the applied potential difference, but the peak is shifted toward larger distances upon increase of the electrode polarization, switching from $1.58 \mathrm{~nm}$ for $0 \mathrm{~V}$ to $1.73 \mathrm{~nm}$ for $9 \mathrm{~V}$. When constant charges simulations are employed, not only is this shift more pronounced, but the peak also becomes much flatter. In the case of the $\mathrm{BMIM}^{+}$cations (right panels), the changes concern the first intense peak, which also becomes less intense and flatter in the case of the constant charge simulations. Finally, we observe that the differences in the structural properties become more visible when the electrodes are highly charged. A comparison of the full ionic density profiles for an applied potential difference of $9.0 \mathrm{~V}$ or a constant charge density of $1 \mathrm{e} \mathrm{nm}^{-2}$ (Figure 2) clearly shows that most of the oscillatory profile close to the interfaces is lost in the second case.

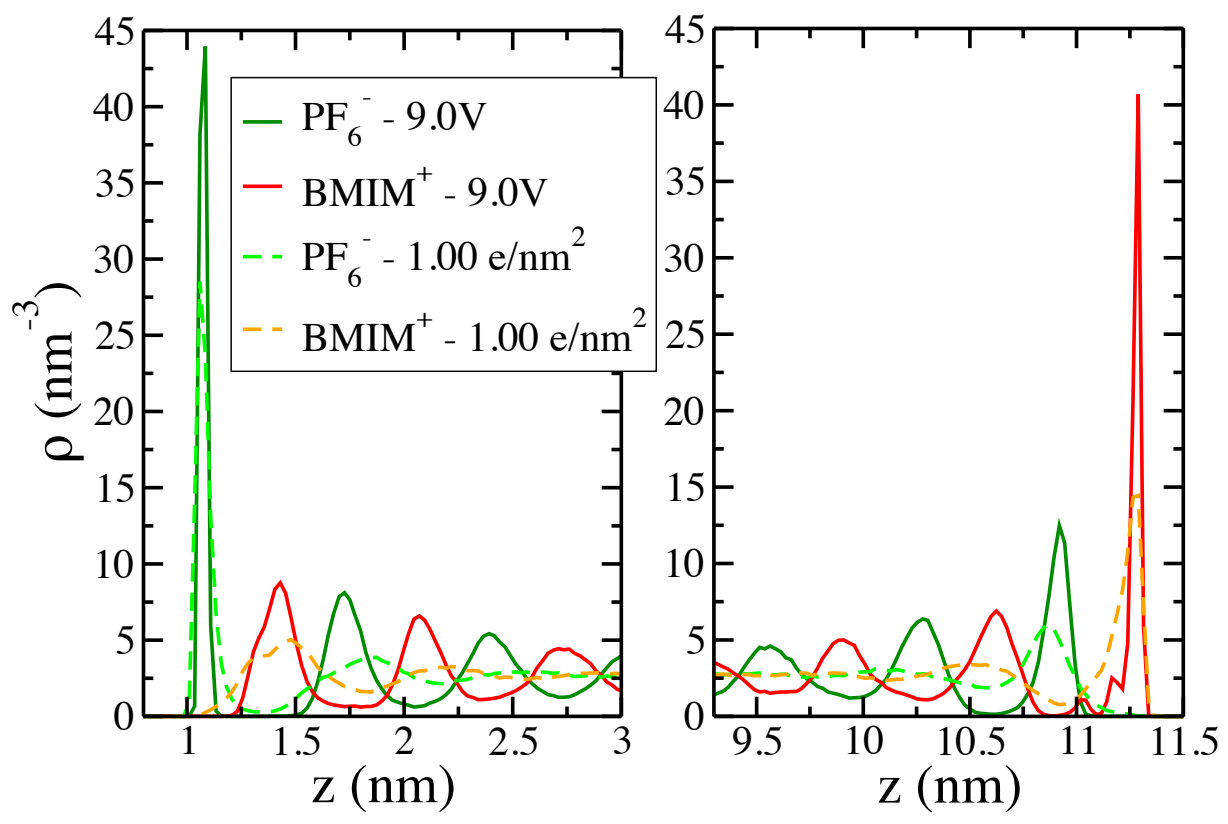

Figure 2: Ionic density profiles for the $9.0 \mathrm{~V}$ constant applied potential $\left(V^{+}=-V^{-}=4.5 \mathrm{~V}\right)$ and the $1 \mathrm{e} \mathrm{nm}^{-2}$ constant charge simulations. Left: positive electrode, right: negative electrode. Similar plots for other electrode potentials are provided in the Supplementary Information.

The difference between these density profiles is due to the fact that in the second case, the constant charge on the electrode constrains the liquid to adopt a given structure. When a constant 
potential is applied, there is an interplay between the charge on the electrode and the local structure of the electrolyte layer facing it, which is therefore allowed to change more smoothly. It is worth mentioning that the variation of the charge with respect to the potential drop at the electrode/electrolyte interface obtained with the constant charge simulations agrees very well with that obtained with the constant potential simulation ones, resulting in similar values for the differential capacitance (data provided in the Supplementary Information). This result may however be partly fortuitous, and should not be taken as a general rule.

The performance of supercapacitors in terms of power (i.e how fast the charge can be delivered) can be assessed by modelling the polarization relaxation during transient regimes. This is done by performing simulations where the applied potential difference or the charge is suddenly changed and by characterizing the processes which occur before the new steady-state is reached ${ }^{17,18}$ (note that the objective of such simulations is not to mimic the cyclic voltammetry setups, in which the potential is applied with a slow scan rate, but rather to study the relaxation phenomena which occur in a supercapacitor under operation). The difference between constant potential and constant charge simulations is then much more spectacular. Although it is already visible in the case of graphite electrode systems, we have picked a more illustrative example by simulating a supercapacitor with nanoporous carbide-derived carbon electrodes. ${ }^{3}$ We have performed 3 different simulations, starting from the same initial configuration. The latter was extracted from a trajectory obtained with uncharged electrodes, and presents the particularity to have some empty pores. The two first simulations were performed with constant applied potential differences of $1.0 \mathrm{~V}$ and 5.0 V respectively, while the third one was performed with a constant charge of 0.01 e (respectively -0.01 e) assigned to each carbon atom of the left (right) electrode: This value corresponds to the average of the fluctuating charges carried by the carbon atoms for a $1 \mathrm{~V}$ applied potential simulation. ${ }^{3}$

As shown on Figure 3, after only 5 ps, the empty pore is already filled for the constant charge simulation. This is not the case for any of the constant potential ones, for which 100 ps is not sufficient to obtain a full charge (only the $1.0 \mathrm{~V}$ situation is shown on Figure 3 since the $5.0 \mathrm{~V}$ one 


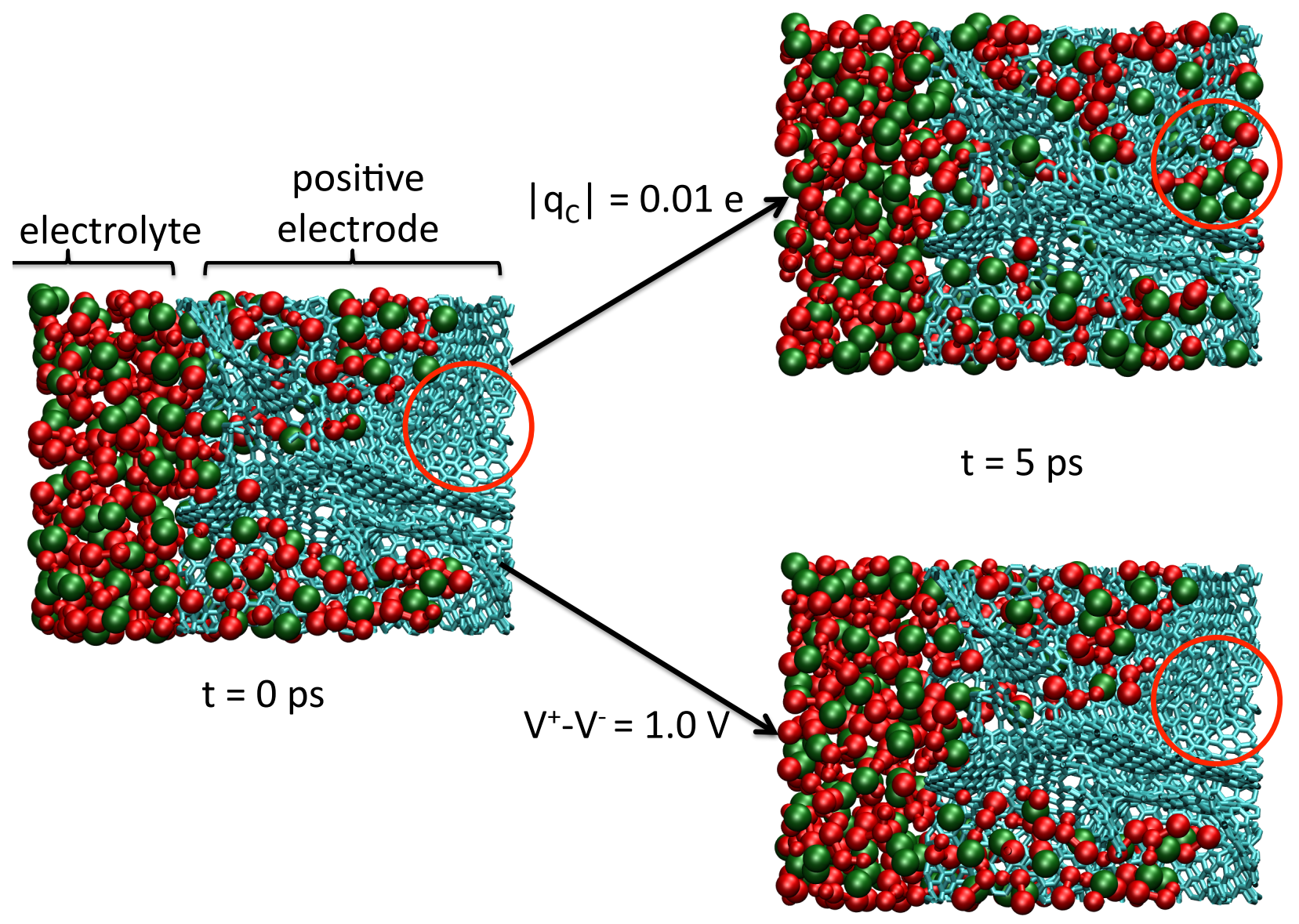

Figure 3: Evolution of the structure inside the positive electrode upon application of a constant charge (top) or a constant applied potential (bottom) simulation. Only part of the simulation cell is shown here. The initially empty pore region is highlighted with a red circle. Green spheres: anions, red spheres: cations.

yields a similar result). Such a difference in the dynamics was already reported by Vatamanu et al. for planar electrodes. These authors concluded that constant potential simulations provided much more reasonable double layer relaxation times. ${ }^{14}$ Here, even more striking is the increase in the temperature due to the Joule effect, as shown in Figure 4. In the constant potential simulations, the calculated heating rate is of $0.08 \mathrm{~K} \mathrm{ps}^{-1}$ (respectively $2.1 \mathrm{~K} \mathrm{ps}^{-1}$ ) for a constant applied potential of $1.0 \mathrm{~V}$ (respectively $5.0 \mathrm{~V}$ ), in very good agreement with a basic estimation based on Ohm's law (see the Supplementary Information for details) of $0.10 \mathrm{~K} \mathrm{ps}^{-1}$ (respectively $2.5 \mathrm{~K} \mathrm{ps}^{-1}$ ). In contrast, in the constant charge simulations the temperature reaches $25,000 \mathrm{~K}$ in $1.0 \mathrm{ps}$, reflecting an extreme energy dissipation and the driving of the system far from equilibrium, with a strong 
macroscopic electric field across the cell. The relaxation processes observed during the latter simulations are therefore unrealistic and this procedure should be avoided for the study of transient regimes occurring in supercapacitors at the molecular scale. Nevertheless, the constant potential simulations with nanoporous carbon electrodes are very expensive from the computational point of view, so that constant charge simulations remain very useful in the first stages of equilibration of the simulation cells. In the case of constant applied potential simulations, the charge on the electrode atoms varies in response to the ionic liquid; as a consequence the charging of the initially empty pores occurs gradually while they become wetted, resulting in much slower relaxation times.

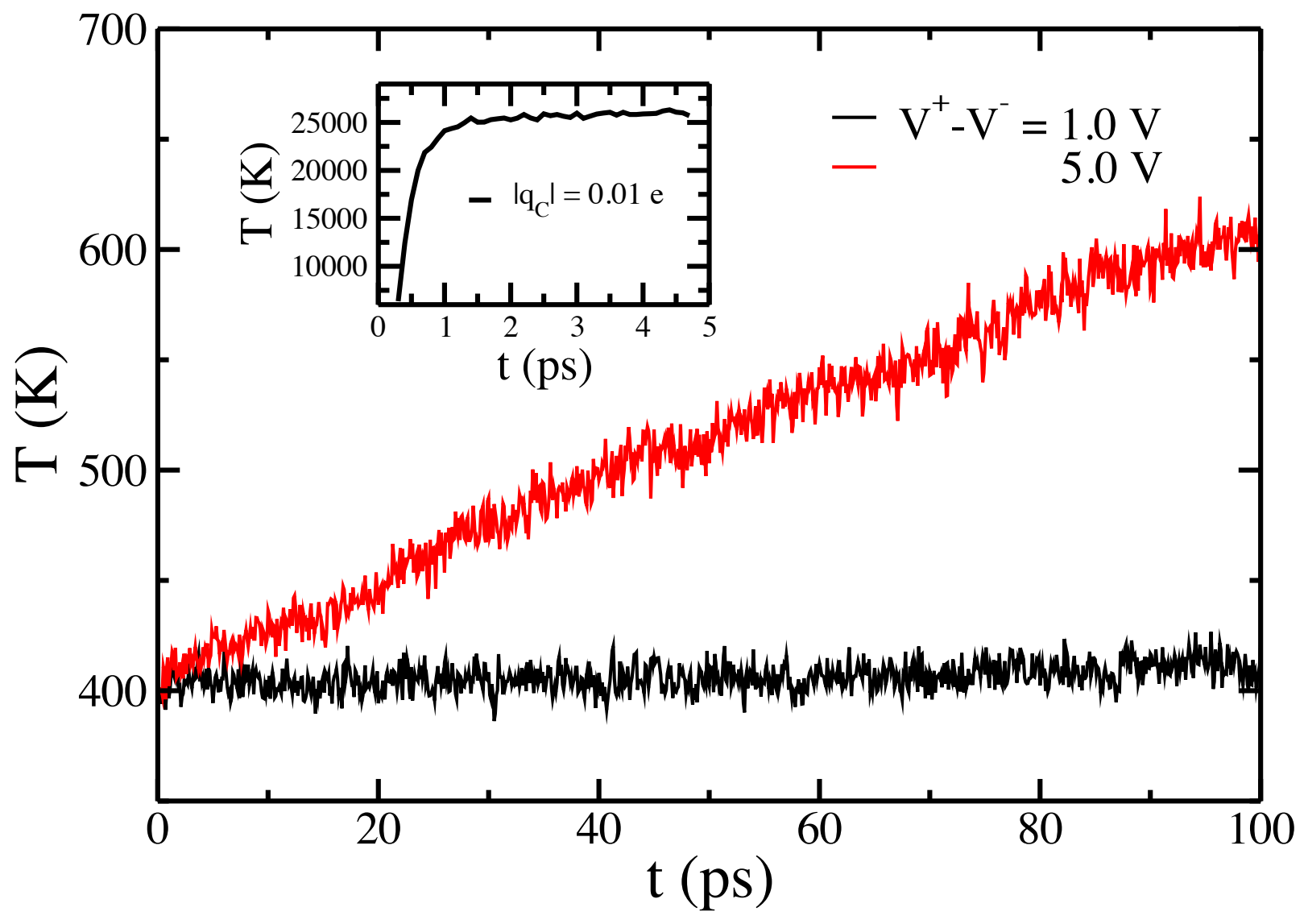

Figure 4: Temperature drift when a constant potential difference is suddenly applied (insert: when a constant charge is assigned to each carbon atom).

In conclusion, we have showed that the use of constant charge simulation setups for modelling supercapacitors based on ionic liquid electrolytes and carbon electrodes alter both the structure of 
the adsorbed fluid at the interface and the timescales over which relaxation phenomena occur. The situation is much more critical for the latter, which are modified by orders of magnitude, but the changes in the structure are also visible and they increase with the charge of the electrode, which may lead to modifications in the thermodynamic properties of the systems. This situation can be paralleled with the role played by ion polarization effects in bulk ionic liquids and molten salts: In the latter case, it is now well known that the simulations performed in the framework of rigid ion models provide a correct description of the structure of singly-charged ions based liquids, but, as soon as multivalent ions are added, such models are not able to yield the complex structural patterns which are observed and it is necessary to resort to a polarizable ion model. ${ }^{28-31}$ Note that here the use of more complex force fields for the ionic liquids, which include polarization effects either explicitly or effectively ${ }^{32,33}$ (and even the electron density breathing effects ${ }^{34}$ ), would also enhance the description of the system. Finally the dynamics is highly affected, and transport properties are usually underestimated by one order of magnitude with rigid ion models. ${ }^{29-31}$ Here the effect is opposite, since constant charge simulations greatly enhance the dynamics of the system. It is therefore important to use constant applied potential conditions when modelling electrodes, despite the associated increase in computational cost. This recommendation is likely to hold for the modelling of systems other than supercapacitors which involve a metal / ionic liquid interface; ${ }^{35}$ for example the role of polarization on the stabilization of metallic nanoparticles in ionic liquids has recently been demonstrated. ${ }^{36}$ It is worth noting that schemes alternative to the selfconsistent calculation of the charges have been proposed, ${ }^{37}$ which allow to decrease significantly this computational time, but these methods have yet to be adapted to the case of porous electrodes.

\section{Computer simulation details}

Following our previous works, the coarse-grained model of Roy and Maroncelli, which yields the correct structural, thermodynamic and dynamic properties of $\mathrm{BMIM}_{-} \mathrm{PF}_{6}$, was used for the ionic liquid. ${ }^{38}$ In all the simulations, the Coulomb interactions are calculated through a two-dimensional 
Ewald summation $^{12,39}$ and a time step of $2 \mathrm{fs}$, which ensures a good conservation of the total Hamiltonian, is used to integrate the equations of motion. For the ionic liquid-graphite simulations, the system consists of 320 ion pairs sandwiched between two groups of three (respectively one) graphite layers for the constant potential (respectively charge) simulations. In the latter case, one plane only was used in order to match with previous studies. The lengths in $x$ and $y$ directions are chosen to match with the lattice parameters of graphite and are equal to 3.22 and $3.44 \mathrm{~nm}$, respectively. The length in the $z$ direction is adjusted to recover the bulk density of the ionic liquid at $400 \mathrm{~K}$ leading to $L_{z}=12.32 \mathrm{~nm}$. For each point, the system is equilibrated during 200 ps before gathering data during $1 \mathrm{~ns}$ in the NVT ensemble. For the ionic liquid-porous carbon simulations, the system consists of 600 ion pairs sandwiched between two porous electrodes ${ }^{40}$ containing 3649 atoms each, as explained in reference. ${ }^{3}$ The length in $x$ and $y$ directions are both equal to $4.37 \mathrm{~nm}$, while the length in the $z$ direction is of $18.64 \mathrm{~nm}$. The systems are simulated during $100 \mathrm{ps}$ in the NVE ensemble, starting from an initial configuration as explained in the main text. Note that the number of simulated ions was enough to keep a constant density in the bulk region during all the simulations.

\section{Acknowledgements}

We acknowledge the support of the French Agence Nationale de la Recherche (ANR) under grant ANR-2010-BLAN-0933-02 ('Modeling the Ion Adsorption in Carbon Micropores'). The research leading to these results has received funding from the European Research Council under the European Union's Seventh Framework Programme (FP/2007-2013) / ERC Grant Agreement n. 102539. We are grateful for the computing resources on JADE (CINES, French National HPC) obtained through the project x2012096728. This work made use of the facilities of HECToR, the UK's national high-performance computing service, which is provided by UoE HPCx Ltd at the University of Edinburgh, Cray Inc and NAG Ltd, and funded by the Office of Science and Technology through EPSRC's High End Computing Programme. 


\section{Supplementary information}

Additional methodology details and supplementary figures are all given in the Supplementary Information. Two movies showing the trajectories used for Figure 3 are also provided. Finally, in a third movie we show the full trajectory (100 ps) for the $1 \mathrm{~V}$ constant applied potential simulation.

\section{References}

(1) Simon, P.; Gogotsi, Y. Materials for Electrochemical Capacitors. Nature Mater. 2008, 7, 845854.

(2) Kondrat, S.; Georgi, N.; Fedorov, M. V.; Kornyshev, A. A. A Superionic State in Nano-Porous Double-Layer Capacitors: Insights from Monte Carlo Simulations. Phys. Chem. Chem. Phys. 2011, 13, 11359-11366.

(3) Merlet, C.; Rotenberg, B.; Madden, P. A.; Taberna, P.-L.; Simon, P.; Gogotsi, Y.; Salanne, M. On the Molecular Origin of Supercapacitance in Nanoporous Carbon Electrodes. Nature Mater. 2012, 11, 306-310.

(4) Wu, P.; Huang, J.; Meunier, V.; Sumpter, B. G.; Qiao, R. Voltage Dependent Charge Storage Modes and Capacity in Sub-nanometer Pores. J. Phys. Chem. Lett. 2012, 3, 1732-1737.

(5) Vatamanu, J.; Borodin, O.; Smith, G. D. Molecular Insights into the Potential and Temperature Dependences of the Differential Capacitance of a Room-Temperature Ionic Liquid at Graphite Electrodes. J. Am. Chem. Soc. 2010, 132, 14825-14833.

(6) Vatamanu, J.; Cao, L.; Borodin, O.; Bedrov, D.; Smith, G. D. On the Influence of Surface Topography on the Electric Double Layer Structure and Differential Capacitance of Graphite/Ionic Liquid Interfaces. J. Phys. Chem. Lett. 2011, 2, 2267-2272.

(7) Chmiola, J.; Yushin, G.; Gogotsi, Y.; Portet, C.; Simon, P.; Taberna, P. L. Anomalous Increase in Carbon Capacitance at Pore Sizes Less Than 1 Nanometer. Science 2006, 313, 1760-1763. 
(8) Chmiola, J.; Largeot, C.; Taberna, P.-L.; Simon, P.; Gogotsi, Y. Desolvation of Ions in Subnanometer Pores and Its Effect on Capacitance and Double-Layer Theory. Angew. Chem., Int. Ed. 2008, 47, 3392-3395.

(9) Wang, H.; Koster, T. K. J.; Trease, N. M.; Segalini, J.; Taberna, P. L.; Simon, P.; Gogotsi, Y.; Grey, C. P. Real-Time NMR Studies of Electrochemical Double-Layer Capacitors. J. Am. Chem. Soc. 2011, 133, 19270-19273.

(10) Richey, F. W.; Elabd, Y. A. In Situ Molecular Level Measurements of Ion Dynamics in an Electrochemical Capacitor. J. Phys. Chem. Lett. 2012, 3, 3297-3301.

(11) Siepmann, J.; Sprik, M. Influence of Surface-Topology and Electrostatic Potential on Water Electrode Systems. J. Chem. Phys. 1995, 102, 511-524.

(12) Reed, S. K.; Lanning, O. J.; Madden, P. A. Electrochemical Interface between an Ionic Liquid and a Model Metallic Electrode. J. Chem. Phys. 2007, 126, 084704.

(13) Pounds, M.; Tazi, S.; Salanne, M.; Madden, P. A. Ion Adsorption at a Metallic Electrode: an Ab Initio Based Simulation Study J. Phys.: Condens. Matter 2009, 21, 424109.

(14) Vatamanu, J.; Borodin, O.; Smith, G. D. Molecular Simulations of the Electric Double Layer Structure, Differential Capacitance, and Charging Kinetics for N-MethylNpropylpyrrolidinium Bis(fluorosulfonyl)imide at Graphite Electrodes. J. Phys. Chem. B 2011, 115, 3073-3084.

(15) Merlet, C.; Salanne, M.; Rotenberg, B.; Madden, P. A. Imidazolium Ionic Liquid Interfaces with Vapor and Graphite: Interfacial Tension and Capacitance from Coarse-Grained Molecular Simulations. J. Phys. Chem. C 2011, 115, 16613-16618.

(16) Xing, L.; Vatamanu, J.; Borodin, O.; Bedrov, D. On the Atomistic Nature of Capacitance Enhancement Generated by Ionic Liquid Electrolyte Confined in Subnanometer Pores. J. Phys. Chem. Lett. 2013, 4, 132-140. 
(17) Lanning, O.; Madden, P. A. Screening at a Charged Surface by a Molten Salt. J. Phys. Chem. B 2004, 108, 11069-11072.

(18) Pinilla, C.; Del Pópolo, M. G.; Kohanoff, J.; Lynden-Bell, R. M. Polarization Relaxation in an Ionic Liquid Confined between Electrified Walls. J. Phys. Chem. B 2007, 111, 4877-4884.

(19) Lynden-Bell, R. M.; Del Pópolo, M. G.; Youngs, T. G. A.; Kohanoff, J.; Hanke, C. G.; Harper, J. B.; Pinilla, C. C. Simulation of Ionic Liquids, Solutions and Surfaces. Acc. Chem. Res. 2007, 40, 1138-1145.

(20) Fedorov, M. V.; Kornyshev, A. A. Ionic Liquid near a Charged Wall: Structure and Capacitance of Electrical Double Layer. J. Phys. Chem. B 2008, 112, 11868-11872.

(21) Feng, G.; Zhang, J.; Qiao, R. Microstructure and Capacitance of the Electrical Double Layers at the Interface of Ionic Liquids and Planar Electrodes J. Phys. Chem. C 2009, 113, 45491559.

(22) Kislenko, S.; Samoylov, I.; Amirov, R. Molecular Dynamics Simulation of the Electrochemical Interface between a Graphite Surface and the Ionic Liquid $[\mathrm{BMIM}]\left[\mathrm{PF}_{6}\right]$. Phys. Chem. Chem. Phys. 2009, 11, 5584-5590.

(23) Shim, Y.; Kim, H. J. Nanoporous Carbon Supercapacitors in an Ionic Liquid: A Computer Simulation Study. ACS Nano 2010, 4, 2345-2355.

(24) Si, X.; Li, S.; Wang, Y.; Ye, S.; Yan, T. Effect of Specific Adsorption on the Differential Capacitance of Imidazolium-Based Ionic Liquid Electrolytes. ChemPhysChem 2012, 13, 16711676.

(25) Li, S.; Feng, G.; Fulvio, P. F.; Hillesheim, P. C.; Liao, C.; Dai, S.; Cummings, P. T. Molecular Dynamics Simulation Study of the Capacitive Performance of a Binary Mixture of Ionic Liquids near an Onion-like Carbon Electrode. J. Phys. Chem. Lett. 2012, 3, 2465-2469. 
(26) Paek, E.; Pak, A. J.; Hwang, G. S. A Computational Study of the Interfacial Structure and Capacitance of Graphene in $[\mathrm{BMIM}]\left[\mathrm{PF}_{6}\right]$ Ionic Liquid. J. Electrochem. Soc. 2013, 160, A1A10.

(27) Merlet, C.; Salanne, M.; Rotenberg, B. New Coarse-Grained Models of Imidazolium Ionic Liquids for Bulk and Interfacial Molecular Simulations. J. Phys. Chem. C 2012, 116, 76877693.

(28) Borodin, O. Polarizable Force Field Development and Molecular Dynamics Simulations of Ionic Liquids. J. Phys. Chem. B 2009, 113, 11463-11478.

(29) Bedrov, D.; Borodin, O.; Li, Z.; Smith, G. Influence of Polarization on Structural, Thermodynamic, and Dynamic Properties of Ionic Liquids Obtained from Molecular Dynamics Simulations. J. Phys. Chem. B 2010, 114, 4984-4997.

(30) Schroder, C. Comparing Reduced Partial Charge Models with Polarizable Simulations of Ionic Liquids. Phys. Chem. Chem. Phys. 2012, 14, 3089-3102.

(31) Salanne, M.; Madden, P. A. Polarization Effects in Ionic Solids and Melts. Mol. Phys. 2011, 109, 2299-2315.

(32) Chaban, V. V.; Voroshylova, I. V.; Kalugin, O. N. A New Force Field Model for the Simulation of Transport Properties of Imidazolium-Based Ionic Liquids. Phys. Chem. Chem. Phys. 2011, 13, 7910-7920.

(33) Chaban, V. V.; Prezhdo, O. V. A New Force Field Model of 1-Butyl-3-Methylimidazolium Tetrafluoroborate Ionic Liquid and Acetonitrile Mixtures. Phys. Chem. Chem. Phys. 2011, $13,19345-19354$.

(34) Madden, P. A.; Wilson, M. 'Covalent' Effects in 'Ionic' Systems. Chem. Soc. Rev. 1996, 25, $339-350$. 
(35) Mendonça, A. C. F.; Malfreyt, P.; Pádua, A. A. H. Interactions and Ordering of Ionic Liquids at a Metal Surface. J. Chem. Theory Comput. 2012, 8, 3348-3355.

(36) Pensado, A. S.; Pádua, A. A. H. Solvation and Stabilization of Metallic Nanoparticles in Ionic Liquids. Angew. Chem., Int. Ed. 2011, 50, 8683-8687.

(37) Petersen, M. K.; Kumar, R.; White, H. S.; Voth, G. A. A Computationally Efficient Treatment of Polarizable Electrochemical Cells held at a Constant Potential. J. Phys. Chem. C 2012, 116, 4903-4912.

(38) Roy, D.; Maroncelli, M. An Improved Four-Site Ionic Liquid Model. J. Phys. Chem. B 2010, $114,12629-12631$.

(39) Gingrich, T. R.; Wilson, M. On the Ewald Summation of Gaussian Charges for the Simulation of Metallic Surfaces. Chem. Phys. Lett. 2010, 500, 178-183.

(40) Palmer, J. C.; Llobet, A.; Yeon, S.-H.; Fisher, J. E.; Shi, Y.; Gogotsi, Y.; Gubbins, K. E. Modeling the Structural Evolution of Carbide-Derived Carbons Using Quenched Molecular Dynamics. Carbon 2010, 48, 1116-1123. 